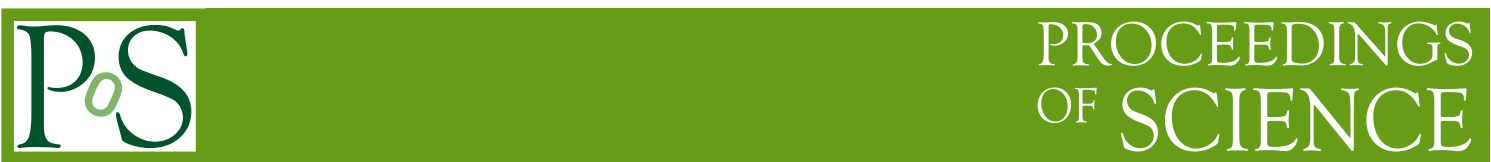

\title{
Modeling Cosmic Dust: How to Use Optical "Constants"
}

\author{
Angela K. Speck* \\ Department of Physics and Astronomy, University of Missouri, Columbia, MO 65211 \\ E-mail: speckan@missouri.edu
}

\begin{abstract}
In order to determine the precise nature of cosmic dust, we use a combination of multi-wavelength ground- and space-based spectroscopy, imaging, laboratory data and modeling. Dust grains scatter, absorb and re-radiate light according to their optical properties, which are sensitive to e.g. the temperature, chemical composition, size, shape, and lattice structure of the dust grains. For example, graphite and diamond are both polymorphs of carbon, and will form under very similar conditions, but their interactions with light are very different. This work provides a primer on how to apply basic physics concepts to understanding how we measure and use the optical properties of candidate cosmic dust species. We discuss the way in which measurements are made, how simplifying assumptions commonly made in astronomy may cause problems and how measurable and calculable parameters from laboratory experiments can be directly or indirectly compared to parameters derived from astronomical observations. Finally, we examine the simplifying assumptions with the most commonly used "synthetic" optical properties for cosmic dust and highlight forthcoming laboratory data as a potential replacement.
\end{abstract}

The Life Cycle of Dust in the Universe: Observations, Theory, and Laboratory Experiments - LCDU 2013, 18-22 November 2013

Taipei, Taiwan

\footnotetext{
* Speaker.
} 


\section{Introduction}

In order to study dust in space, astronomers rely on combining theoretical and observational data with laboratory studies of analog materials. The precise nature of the laboratory investigations cover a wide range of methods, samples and outputs. Frequently, there is a disconnect between the laboratory assumptions and the data to interpret astronomical observations. The purpose of this paper is to provide an introduction to how the optical properties of solids are obtained and how these datasets relate to astronomy.

There are multiple terms and symbols used to describe various ways in which light interacts with solids and frequently the same letters (especially $k$ ). In order to prevent confusion, terms and symbols will be precisely defined.

In $\S$ 2 we will consider how we describe and quantify the various ways in which light interacts with solids; in $\S 3$ we discuss how we analyze astronomical observational data to extract properties of the cosmic dust observed; and in $\S €$ we look at how laboratory measurements (e.g. absorbance and absorptivity) compare to parameters extracted from astronomical observations (e.g. optical depth, absorption/extinction efficiency). In $\S \S$ we discuss how laboratory data are used to extract more intrinsic properties (e.g. complex refractive index) in order to be used in modeling astronomical enivronments; in $\S$ we show comparisons of spectra derived from laboratory spectra of bulk and particulate samples as well as calculations that assume spherical and non-spherical grains. Finally, in $\S$ \e discuss the most commonly used optical constants (complex refractive indices) and we discuss the simplifying and erroneous assumptions in the most commonly used optical constants datasets and how we can improve upon them.

\section{How light interacts with solids}

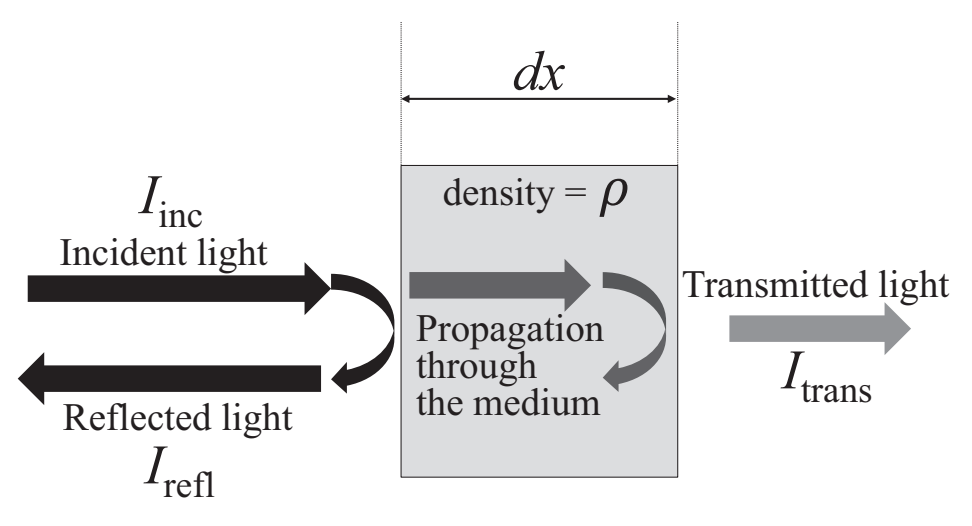

Figure 1: Light interacting with a parallel-faced slab of solid material.

Consider light entering a slab of solid material with parallel faces as shown in Fig. 1. Incident light hits the face of the slab; some light is transmitted and some light is reflected. Assuming the material is perfectly homogeneous, the light will propagate through the medium until it reaches the other face, at which point some light will be reflected and some will be transmitted. In addition, as the light propagates through the material, some light may be absorbed. 
Transmittance, $T^{*}$ is defined as the ratio of the intensity of transmitted ( $\left.I_{\text {trans }}\right)$ and incident $\left(I_{\text {inc }}\right)$ light, i.e. $I_{\text {trans }} / I_{\text {inc }}$. Similarly reflectivity, $R$ and absorptivity, $A$ are the ratios of absorbed to incident light and reflected to incident light, respectively. Since all light incident on the slab must be either transmitted, reflected, or absorbed, we get:

$$
\begin{aligned}
& I_{\text {inc }}=I_{\text {trans }}+I_{\text {refl }}+I_{\text {abs }} \\
& \frac{I_{\text {trans }}}{I_{\text {inc }}}=1-\frac{I_{\text {refl }}}{I_{\text {inc }}}-\frac{I_{\text {abs }}}{I_{\text {inc }}}
\end{aligned}
$$

Therefore,

$$
T=1-R-A
$$

Note that all these properties are wavelength dependent.

While factually correct, Eq. 2.2 presents an over simplified view of the process. Looking at Fig. 1 1 we can see that reflection at the second surface must be neglible for Eq. 2.2 to hold. The problem is demonstrated in Fig. 2, which shows that the measured values and $T$ and $R$ will be strongly affected by back-reflections. In particular the absorption of the light reflected from the back of the sample as it transits towards the front will affect the measurements of both $T$ and $R$ and thus the inferred values of $A$.

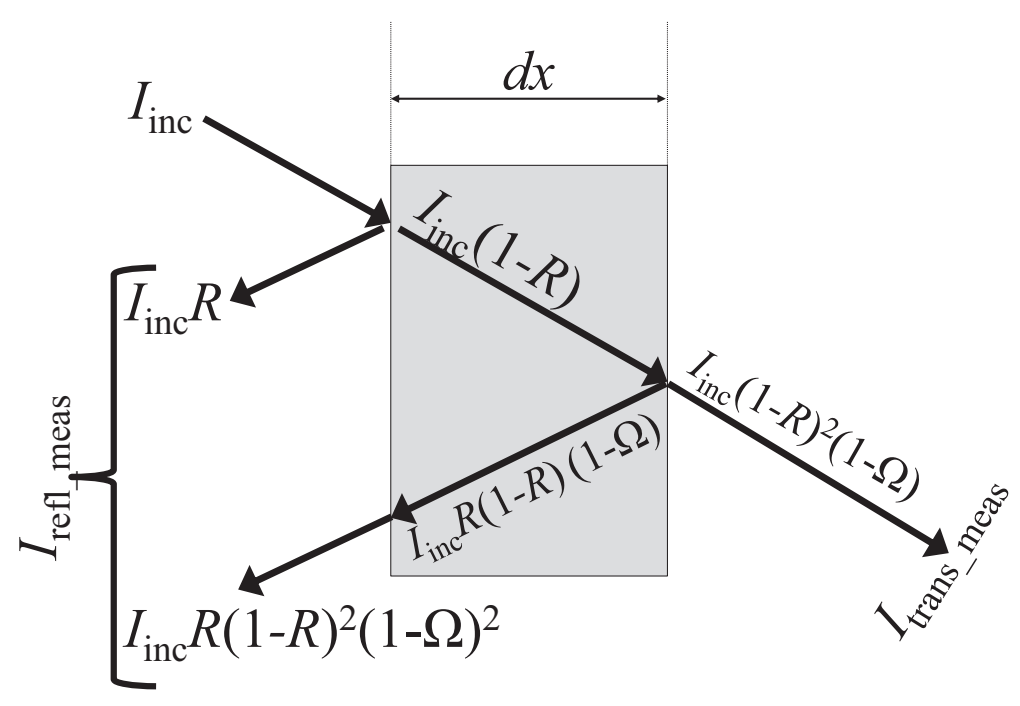

Figure 2: Light interacting with a parallel-faced slab of solid material: the effect of internal reflections. The reflectance is $R$ as defined above. The amount of light absorbed by a single passage through the slab is denoted by $\Omega$. $I_{\text {trans_meas }}$ is the amount of transmitted light that gets measured, while $I_{\text {refl_meas }}$ is the amount of reflected light measured.

In addition, there are several phenomena that can affect the direct transmission of light, as shown in Fig. 3. During propagation of light through a medium, light can be refracted, absorbed, or scattered. These processes will affect the light collected by detectors in the laboratory.

*which is also referred to as transmissivity [1] 


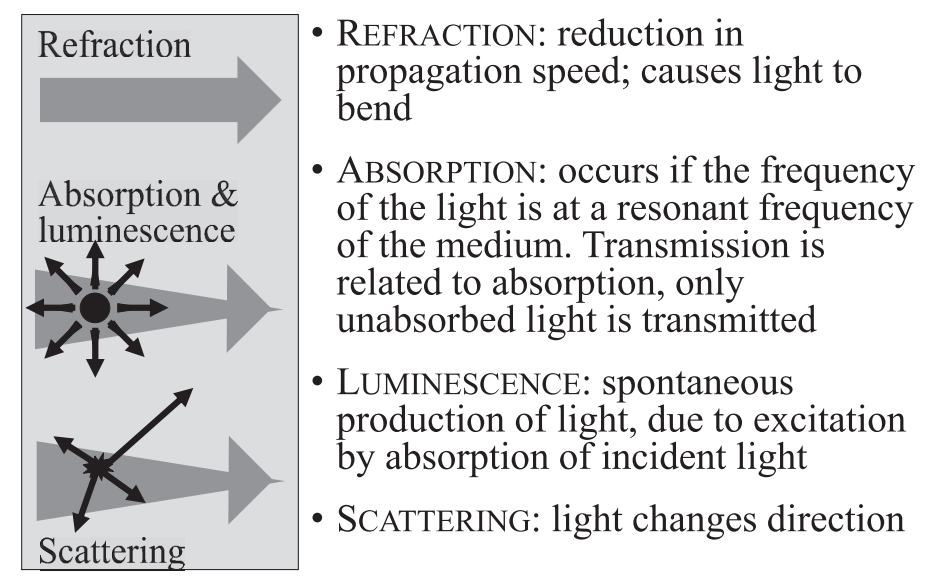

Figure 3: Light interacting with a parallel-faced slab solid (part 3.

Let us now consider the opacity or absorption properties of the solid. In Fig. 1, the incident flux $I_{\text {inc }}$ is diminished by the passage of the light according to the absorptive properties of the medium. Now we can define the light lost by interacting with the medium as $d I_{\lambda}$, such that:

$$
d I_{\lambda}=I_{\text {inc }}-I_{\text {trans }}
$$

But the amount of light absorbed/transmitted depends on the thickness of the slab of medium, $d x$, (and the wavelength, $\lambda$ ) so that:

$$
d I_{\lambda}=-k_{\lambda} \rho I_{\lambda}(x) d x
$$

where $k_{\lambda}$ is the opacity or sometimes called the mass absorption coefficient, measured in area per unit mass, and $\rho$ is the mass density of the medium.

$$
I_{\lambda}(x)=I_{\lambda}(0) e^{-k_{\lambda} \rho x}
$$

Flux decreases exponentially with penetration into a medium. This is the Beer-Lambert Law. Now we can combine the opacity $k_{\lambda}$ with the density $\rho$ to get "extinction coefficient" or sometimes just absorption coefficient ${ }^{\dagger} \alpha=-k_{\lambda} \rho$ so that Eq. 2.3 becomes

$$
I_{x}=I_{\text {inc }} e^{-\alpha x}
$$

In fact, we need to combine Eq.2.2 with Eq. 2.3 and the scenario depicting back reflection from the second surface of the medium as shown in Fig. I In this case the transmitted light is given by:

$$
T=\left(1-R_{1}\right) e^{-\alpha x}\left(1-R_{2}\right)
$$

Assuming that the surface reflections are identical, $R_{1}=R_{2}=R$, we get:

$$
T=(1-R)^{2} e^{-\alpha x}
$$

\footnotetext{
†but NOT mass absorption coefficient!
} 
Assuming that reflections at surfaces are negligible (or can be eliminated by using slabs of differing thicknesses, see [-1]) and combining Eq. 2.4 with out definition of transmittance $T$ (from the beginning of $\S$ ) we get

$$
T=e^{-\alpha x}=e^{-a}
$$

Absorbance, $a$, is the exponent in the decay of light intensity due to absorption: $a=\alpha x=k_{\lambda} \rho x$, where $\alpha$ is extinction coefficient, $k_{\lambda}$ is the opacity (or mass absorption coefficient) and $\rho$ is the mass density of the medium. Optical depth $\tau_{\lambda}$ of an absorbing material is defined by:

$$
I_{x}=I_{\text {inc }} e^{-\tau_{\lambda}}
$$

From equations 2.4. 2.6 and 2.7 we see that the absorbance $a$ is similar to optical depth $\tau_{\lambda}$. In order to compare astronomical data in which we have a wavelength-dependent optical depth we can use absorbance after accounting for surface reflections. Now we can relate the absorbance $(a)$ and absorptivity $(A)$ via equations 2.1, 2.2 and 2.6:

$$
A=1-e^{-a}
$$

To summarize these measurable optical properties from lab experiments:

$$
\begin{gathered}
\text { Absorptivity, } A=\frac{I_{\mathrm{abs}}}{I_{\mathrm{inc}}} \\
\text { Absorbance, } a=\alpha x=k_{\lambda} \rho x \\
A=1-e^{-a} \\
T=(1-R)^{2} A
\end{gathered}
$$

\section{Modeling Astronomical Observations}

A spectrum can be represented by a sum of the fluxes contributing to it. For a system containing a star or even a group of stars the spectrum can be broadly described by:

$$
F_{\text {total }}(\lambda)=F_{*}(\lambda)+F_{\text {dust }}(\lambda)
$$

A star can usually be approximated by a blackbody with a temperature $T_{\star}$ whereas the dust is made up of contributions from dust grains of difference sizes, shapes, compositions and crystal structures. The flux from an individual dust grain is a product of the Planck curve $\left(B_{\lambda, T}\right)$ for the temperature $T$ of the individual dust grain and an absorption efficiency factor $Q_{\lambda}$ for a grain of that size, shape, composition and crystal structure.

Astronomers often consider a simplified case in which the spectrum, $F_{\text {total }}(\lambda)$, can be interpreted as a product of the underlying continuum and an absorption efficiency factor $Q_{\lambda}$ for the entire spectrum (e.g., [3]). Then:

$$
F_{\text {total }}(\lambda)=Y \times Q_{\lambda} \times B_{\lambda}(T)
$$

where $B_{\lambda}(T)$ is the Planck function for a black body of temperature $T$; $Q_{\lambda}$ is a composite value including contributions from all dust grains of various sizes, shapes, crystallinities and compositions; 
and $Y$ a scale factor that depends on the number of dust particles, their geometric cross section and the distance to the star.

Using a simple continuum-divided spectrum assumes the type of spectrum described by Equation 3.2. Dividing the entire spectrum by the fitted underlying continuum leaves only the absorption efficiency $\left(Q_{\lambda}\right)$ of the dust (assuming all dust species have the same blackbody temperature). The continuum-divided spectrum is effectively an optical depth spectrum (c.f. Eq. 2.7) which is due to the absorption efficiency for a composite grain that will represent all of the grains present.

In reality the spectrum should be represented by:

$$
F_{\lambda}=F_{\star}+\sum_{i=1, j=1}^{n, m} Y_{j} \times Q_{\lambda, j} \times B_{\lambda, i}\left(T_{i}\right)
$$

where each $B_{i}$ represents a single dust (or stellar) temperature black body (of which there are $n$ in total), each $Q_{j}$ represents the absorption efficiency for a single grain type as defined by its size, shape, composition and crystal structure and $Y_{j}$ is a scale factor that depends on the number of dust particles, their geometric cross section and the distance to the star. In this case, we subtract the stellar flux $\left(F_{\star}\right)$ and are left with:

$$
F_{\text {dust }}=\sum_{i=1, j=1}^{n, m} Y_{j} \times Q_{j} \times B_{i}
$$

For optically thin cases, we often then assume the dust is dominated by a single temperature (c.f. 沺) so that

$$
F_{\text {dust }} \propto Q_{\lambda} \times B_{\lambda}(T)
$$

which is very similar to our original simple model for the dust emssion as shown in Eq. 3.2.

Now that we have a way to extract either optical depth or absorption efficiencies ( $Q$-values) from observations we need to compare with laboratory data.

\section{Comparing Laboratory and Astronomy properties of dust}

To compare laboratory data with astronomical observations we need to extract a version of the laboratory data that is comparable to the absorption efficiency, $Q$-value. To get this we need to consider how $Q$-values are defined. For a non-blackbody dust grain we define an absorption cross-section $\sigma_{\mathrm{abs}}$ as the effective geometrical cross-section of the particle once we account for it not being a blackbody:

$$
\sigma_{\mathrm{abs}}=Q_{\mathrm{abs}} \times \Upsilon
$$

where $\Upsilon$ is the geometrical cross-sectional area of a dust grain. Now if we consider how the absorption cross section gives rise to absorption we get:

$$
\frac{I_{\mathrm{abs}}}{I_{\mathrm{inc}}}=A=\sigma_{\mathrm{abs}} n L
$$


where $A$ is the absorptivity (see $\S$ 凤) $n$ is the number density of absorbing particles, and $L$ is the pathlength or thickness of the absorbing zone.

$$
n=\frac{\rho}{M_{\mathrm{mol}} \times m_{H}}
$$

where $M_{\mathrm{mol}}$ is the molar mass of the solid and $m_{H}$ is the mass of a hydrogen atom. Combining equations 4.1 and 4.2 we get:

$$
Q_{\mathrm{abs}}=\frac{A \times M_{\mathrm{mol}} \times m_{H}}{\Upsilon \rho L}
$$

It is clear from equation 4.3 that Q-values $\propto$ absorptivity such that:

$$
\begin{gathered}
Q_{\mathrm{abs}}=\zeta A \text { where } \zeta=\frac{M_{\mathrm{mol}} \times m_{H}}{\Upsilon \rho L} \\
Q_{\mathrm{abs}} \propto A
\end{gathered}
$$

Consequently, while the shape, peak position and FWHM of spectral features shown in $Q$-values will be identical to those for $A$, the absolute values depend on the pathlength and on the crosssection areas of a given grain distribution.

To summarize how laboratory data correspond with astronomical observational data,

$$
\begin{gathered}
\text { Absorption efficiency, } Q_{\lambda} \equiv \text { Absorptivity, } A(\lambda) \\
\text { Optical depth, } \tau_{\lambda} \equiv \text { Absorbance, } a(\lambda)
\end{gathered}
$$

\section{Complex Refractive Indices and Dielectric Functions}

While we can extract optical properties of solids that are directly comparable to observations (i.e, $Q \equiv A$, see Eq.4.4), this is sometimes not what is needed for modeling. In particular, if we want to understand the effect of grain size and shape on the optical properties or if we have a system that contains grains of multiple sizes and temperatures, we want to use other intrinsic optical properties of the slab solids, i.e. the complex refractive index, $m$, and the dielectric function $\hat{\varepsilon}$.

$$
\begin{gathered}
m=n+i k \\
\hat{\varepsilon}=\varepsilon_{1}+i \varepsilon_{2}
\end{gathered}
$$

where $n$, the real part of the complex refractive index, relates to the speed of propagation of light through the medium (i.e. how we first learn about refractive index); and $k$, the imaginary part is related to absorption of light.

The complex refractive index and the complex dielectric function are closely related:

$$
\begin{gathered}
m=\sqrt{\hat{\varepsilon}} \\
\varepsilon_{1}=\Re\left(m^{2}\right)=n^{2}-k^{2}
\end{gathered}
$$




$$
\varepsilon_{2}=\mathfrak{I}\left(m^{2}\right)=2 n k
$$

To understand how the real and imaginary parts of the complex refractive index affect light, let us consider light as an idealized, sinusoidal wave described by:

$$
H=H_{0} e^{-i(\omega t-\kappa x)}
$$

where $H_{0}$ is the initial wave amplitude, $\omega$ is the angular frequency, and $\kappa$ is the wavenumber $(2 \pi / \lambda)$.

Once the light enters a medium, the velocity becomes $v=c / m$ so that:

$$
H=H_{0} e^{-i(\omega t-\kappa m x)}
$$

substituting in for $m$ from Eq. 5.1 we get:

$$
\begin{gathered}
H=H_{0} e^{-i(\omega t-\kappa[n+i k] x)} \\
H=H_{0} e^{-i(\omega t-\kappa n x)} e^{-\kappa k x}
\end{gathered}
$$

The intensity (flux) of the light is proportional to the square of the amplitude, $H$. It is clear from Eq. 5.3 that the intensity will decrease exponentially with a decay constant of $2 \kappa k=4 \pi k / \lambda$

Combining the Beer-Lambert Law (Eq. 2.3) and Eq. 2.4 with the wavefunction of the light traveling through the medium we get the variation in intensity (flux) to be:

$$
\begin{gathered}
F_{\lambda}(x)=H^{2}(x)=H_{0} e^{-i(\omega t-\kappa n x)} e^{-\frac{4 \pi}{\lambda} k x}=I_{\text {inc }} e^{-\alpha x} \\
I_{\text {inc }}=H_{0} e^{-i(\omega t-\kappa n x)} \text { and } \alpha=\frac{4 \pi}{\lambda} k
\end{gathered}
$$

where $\alpha$ is extinction coefficient and $k$ is the imaginary part of the complex refractory index.

$$
T=(1-R)^{2} e^{-a}
$$

Therefore by measuring the transmission and reflection from a sample we can detemine the imaginary part of the complex refractory index, $k$. To get the real part $n$ we need to either use Kramers-Kronig analysis (see [5]) or we can use this relation:

$$
R=\frac{(n-1)^{2}+k^{2}}{(n+1)^{2}+k^{2}}
$$

\section{Comparing laboratory and "theoretical" spectra}

One of the ways in which we use complex refractive indices is to calculate spectra and determine the effect of, e.g., grain shape, size etc. However, sometimes calculated spectra are not precisely the same as those directly measured. For instance, Fig. 4 (left; from [6]) shows the difference between the absorption cross-section $\left(\sigma_{\text {abs }}\right)$ for hematite $\left(\mathrm{Fe}_{2} \mathrm{O}_{3}\right)$ for direct measurements vs 
those calculated using spherical grains (from Mie theory) or continuous distributions of ellipsoids. It is clear from the figure that spherical grains are far from providing a good approximation to reality.

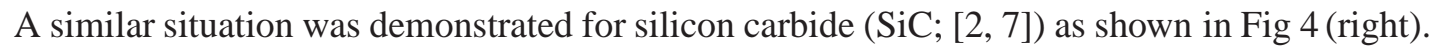
In this case we see the comparison between spectra calculated from laboratory-measured complex refractive indices and those calculated from "synthetic" indices [8]. The "synthetic" data from [8] was produced by compiling and manipulating data from many disparate laboratory studies of SiC and then the complex refractive indices are extracted assuming that the dust grains are spherical. In fact the calculated spectra are only valid if the grains in the model are spherical. Comparing the spectra generated from the synthetic and real refractive indices we see again that they are different. Furthermore, the problem of using spherical grain models is demonstrated. We will investigate the problems of synthetic spectra further in the next section.
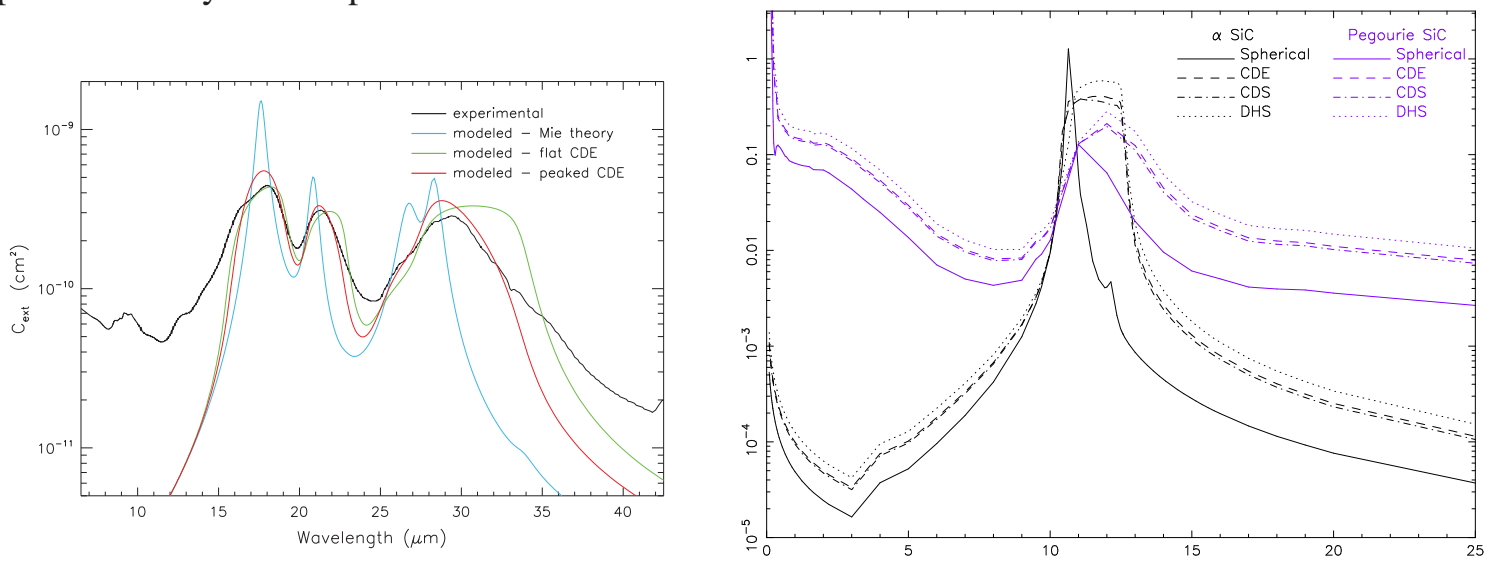

Figure 4: Left: Comparison of absorption cross sections for hematite $\left(\mathrm{Fe}_{2} \mathrm{O}_{3}\right)$ from Marra et al. (2011). The black line labeled experimental is $\sigma_{\mathrm{abs}}$ measured directly from a distribution of small grains of hematite. The colored lines are the $\sigma_{\mathrm{abs}}$ spectra calculated from complex refractive indices for bulk (slab) hematite. Right: Comparison of calculated absorption efficiencies for $\mathrm{SiC}$ using synthetic [8] and laboratory-measured [2] complex refractive indices for $\mathrm{SiC}$. Mie theory $=$ spherical grains; $\mathrm{CDE}=$ Continuous distribution of ellipsoid; CDS = continuous distribution of spheroids; and DHS = distribution of hollow sphere, which is

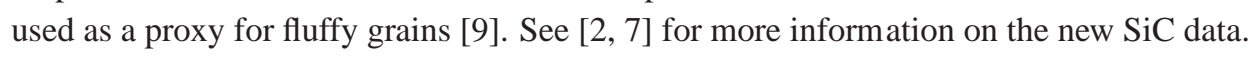

Looking more closely at the left panel in Fig. 4 we see that there is a still a difference between the best model spectrum (with the "peaked" continuous distribution of ellipsoidal grains") and the directly observed $\sigma_{\text {abs }}$ values. There are several reasons for this including scattering from particular material and non-ellipsoidal grain shapes. Looking at Figs. 1, 目 and , we can see that internal scattering in a slab will affect the measurements of transmittance and reflectance and thus any derivation of absorbance, absorptivity or complex refractive indices. Fig. 5 shows how using a distribution of particles has this effect and thus may affect the measurements of $T$ and $R$ and thus the inferred absorption properties. Early laboratory studies compared directly measured spectra with those calulated from the complex refractive indices and found discrepancies. It was suggested that the discrepancies implied the need to correct the measured spectra. However, the discrepancies were due to experiment methods, non-identical samples and non-dilute particle distributions, rather

\footnotetext{
$\$$ "peaked" denoted a distribution in which not all shapes of ellipsoids of equally abundant.There are more grains that are nearly spherical and severaly prolate or oblate.
} 
than an intrinsic difference in the optical functions. It was shown that as long as care is taken with the measurement, identical results can be achieved with particulate and slab samples of the same material [10]

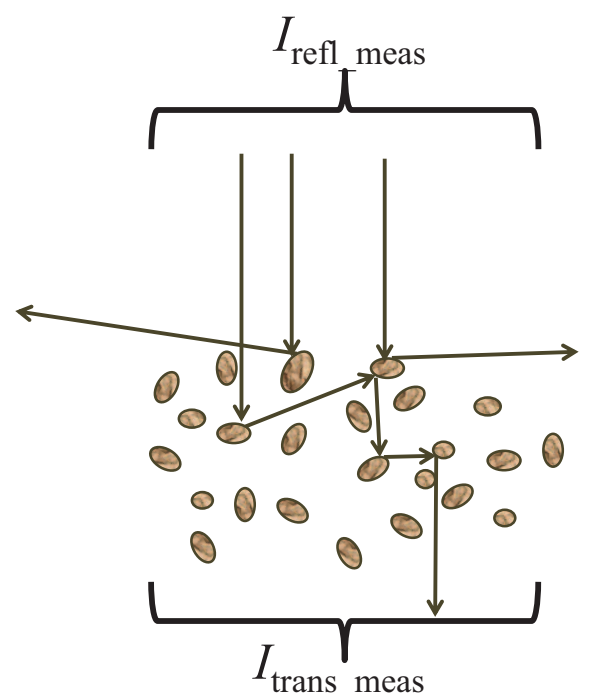

Figure 5: Schematic depiction of the effect measuring reflectance and transmittance from a distribution of particles. Light scattered to the sides is not detected in either $R$ and $T$ measurements and leads to overestimates of absorption

\section{Selecting Optical Constants: A case study of astronomical silicates}

\subsection{A brief history of the classic " $10 \mu \mathrm{m}$ " silicate feature}

One of the most commonly observed features associated with cosmic dust is the so-called " $10 \mu \mathrm{m}$ " silicate feature. It was first observed in the late sixties as an emission feature in the infrared (IR) spectra of several evolved stars [11]. Shortly thereafter it was observed in absorption in the interstellar medium (ISM; [12, 13]). Since then, it has been found in many astrophysical environments including the solar system and extrasolar planetary systems (e.g., [14] and references therein), the circumstellar regions of both young stellar objects and evolved intermediate mass stars (asymptotic giant branch [AGB] stars, and planetary nebulae; e.g., [15, 16]); many lines of sight through the interstellar medium in our own galaxy (e.g., [17, 18]; in nearby and distant galaxies (e.g., [19]).

Initially this feature was attributed to silicate minerals [20] based on spectra of mixtures of crystalline silicate species predicted to form by theoretical models [21, 22]. However, lab spectra of crystalline silicate minerals showed more structure within the feature than observed in the astronomical spectra (see e.g. [23, 24]). Subsequent comparison with natural glasses (obsidian and basaltic glass; from e.g. [25] and with artificially disordered silicates [26, 27] showed that disordered silicate is a better candidate for the $10 \mu \mathrm{m}$ feature than any crystalline silicate mineral.

\subsection{History of laboratory spectra for the $10 \mu \mathrm{m}$ feature}

Since the discovery of the $10 \mu \mathrm{m}$ feature, there have been many laboratory studies producing IR spectra and optical constants of various samples for comparison with and modeling of observational 
data. In addition, "synthetic" complex refractive indices have been derived from observational spectra (based in part on real mineral data) in order to match the observed features (e.g. [28, 29, 30]

The first laboratory spectra used in astronomical silicate studies were of crystalline silicates and natural glasses (e.g., [25]). Various studies have attempted the produced "amorphous" samples through chemical vapor deposition (e.g., [26]), smokes (e.g., [31]), ion-irradiation of crystalline samples (e.g., [27]), and laser ablation of crystalline samples ([32]). Further refinement of laboratory techniques were achieved by the group at Jena (e.g. [33, 34]). For a detailed discussion of these previous laboratory data see [35].

However, most of the laboratory data available comes in the form of mass absorption coefficients or some variation in $Q$-values and frequently have cover only a limited wavelength. In order to incorporate dust species into models of astrophysical environments we need often need the complex refractive indices. Consequently, many astronomers choose to use "synthetic" refractive indices because they cover the large wavelength range. Moreover, laboratory spectra often yield $\sim 10 \mu \mathrm{m}$ features that are not a good match to observed features. Synthetic refractive indices as usually derive in part from observations, and thus can match (some) observations.

\subsection{Synthetic Functions}

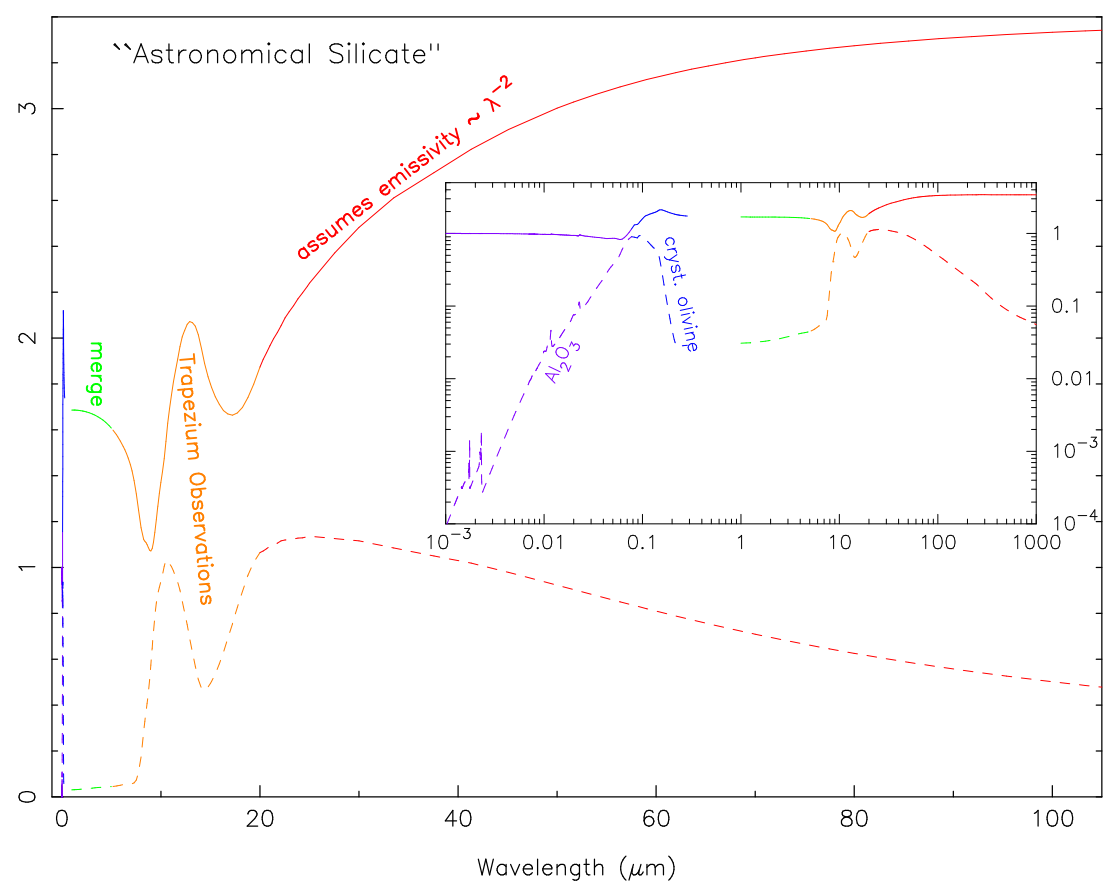

Figure 6: Complex refractive index of "Astronomical Silicate" from [28]. Main figure solid lines $=n$, real part of the complex refractive index; dashed lines $=k$, imaginary part of the complex refractive index. Inset: same as main figure but with logarithmic axes. Purple = far UV to X-ray region, from alumina; Blue = UV-optical, from crystalline olivine; Green - merge between UV-optical measurements of olivine and derivation from observations in the infrared; Orange - derived from infrared observations of the Trapezium; Red - assumes an emissivity index $\beta=2$ based on some data from terrestrial silicates.

Complex refractive indices (otherwise known as optical functions) are extremely important as they are used as inputs for radiative transfer models and in analyzing the effect of grain mor- 
phologies on spectral features. However, quality laboratory data are not available for many of the compounds believed to exist in space, particularly at ultraviolet-visible (UV-vis) and far-infrared wavelengths. Thus, the paucity of data for dust grains is problematic for modeling dusty environments. Both Draine \& Lee (1984) [28] and Ossenkopf, Mathis \& Henning (1992) [29] compiled observational spectral data and derived dust opacities from which they calculated new optical functions specifically designed to match the astronomical observations. More recently, Draine (2003) [30] updated the Draine \& Lee optical function, but the major problems remain. The assumptions implicit in those studies yield optical functions that will match some spectral features, and can be used for comparison of optical depths between different dusty environments, they do not provide information on the true nature of the dust in space or how it varies from location to location. These calculated optical constants fail to match many observed features (e.g. [36, 37])

In both cases, the optical functions are generated by compiling data from a number of sources in order to get the broad wavelength coverage needed. The region around the classic $10 \mu \mathrm{m}$ feature is based on astronomical observations rather than laboratory data. For Draine \& Lee, the $10 \mu \mathrm{m}$ region was determined from observations of the Trapezium. Meanwhile, Ossenkopf et al. used the mean $10 \mu \mathrm{m}$ feature from $\sim 500$ evolved red giants from [38]. In all cases ([28, 29, 30]) the profile of the $10 \mu \mathrm{m}$ feature comes from observations and thus cannot be used to diagnose differences in silicate features from place to place. Figure 6 shows how the data in [30] and [28] were compiled. For the $\mathrm{x}$-ray region, the data comes from laboratory experiments on $\mathrm{Al}_{2} \mathrm{O}_{3}$ (not amorphous silicate.). For the UV portion, the data comes from laboratory experiments using crystalline (not amorphous) silicate. The near-infrared regions is simply a merge between the adjacent optical and mid-IR regions, while the mid-IR comes from astronomical, not laboratory observations. Finally, the far-infrared to radio region was determined assuming an emissivity law $(\beta=2)$. As will be seem in other papers in this volume, the value of $\beta$ is not so simple and depends and many factors including composition, crystal structure, grain size and temperature (see also [39, 40]. Figure 7 shows the real ( $n$, top panel) and imaginary $(k$, bottom panel) parts of complex refractive indices from the most commonly used synthetic optical functions [28, 29, 30] together with new lab-based data [41]. The new optical functions are measure directly from a single sample of iron-free silicate with otherwise solar abundances (see [35]) over a large wavelength range. It is clear that the synthetic optical functions have significantly different profiles to this new "real" dataset which will soon be publicly available[41].

\section{Acknowledgments}

I would like to thank my longstanding collaborators without whom I would not understand this stuff - Anne Hofmeister, Karly Pitman and Alan Whittington. I would also like to thank my students past and present, grads and undergrads, who have regularly indulged my need to explore these topics and give me an opportunity to use them as a test audience.

\section{References}

[1] Fox, M. 2002 "Optical Properties of Solids" Oxford University Press. 

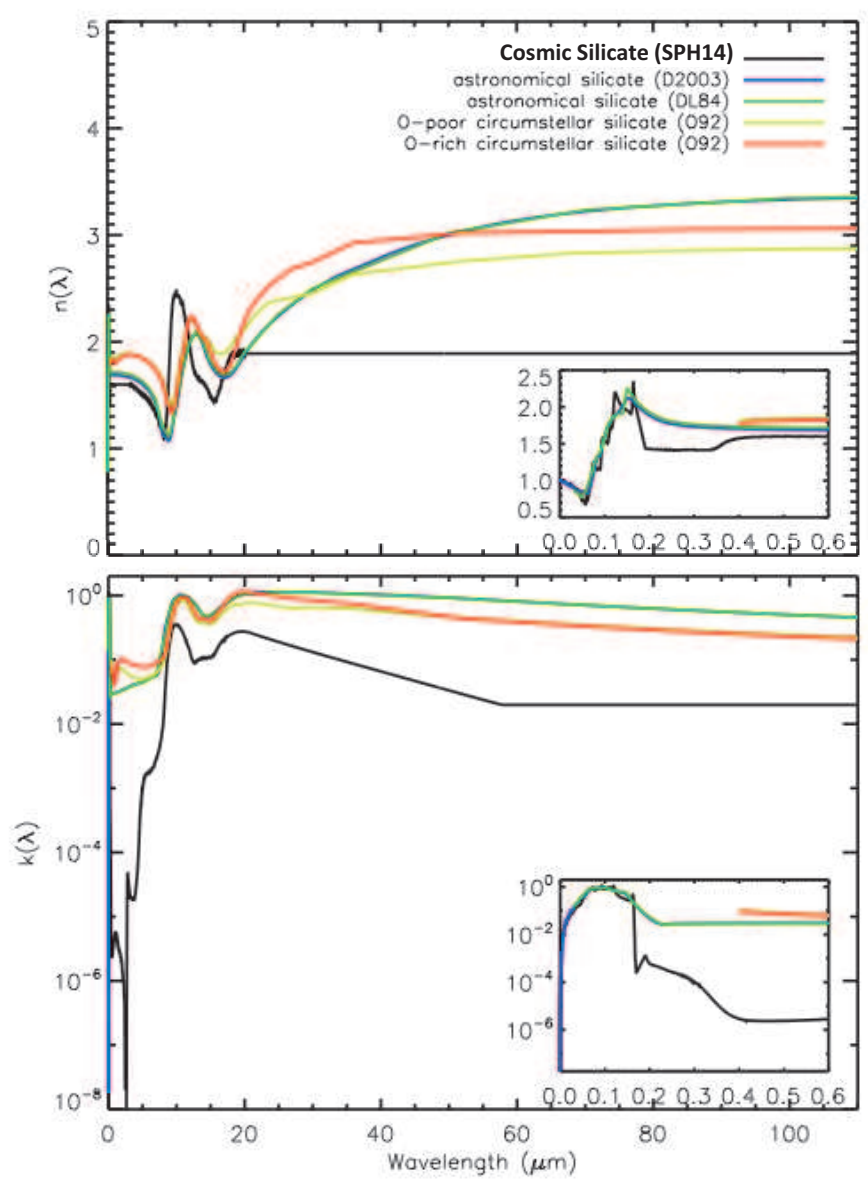

Figure 7: Comparison of synthetic complex refractive indices for "astronomical silicate" from [28, 29] with laboratory-derived optical function of a cosmic abundance silicate from [41] (details of the infrared portion and the sample synthesis available in [35].

[2] Hofmeister, A. M., Pitman, K. M., Goncharov, A. F., Speck, A. K. Optical constants of silicon carbide for astrophysical applications. II. Extending optical functions from IR to UV using single-crystal absorption spectra 2009, ApJ, 696, 1502.

[3] Speck, A. K., Barlow, M. J., Skinner, C. J., , The nature of the silicon carbide in carbon star outflows 1997, MNRAS, 288431.

[4] Guha Niyogi, S., Speck, A. K., Onaka, T., A Temporal Study of the Oxygen-rich Pulsating Variable Asymptotic Giant Branch Star, T Cep: Investigation on Dust Formation and Dust Properties 2011, ApJ, 733, 93.

[5] Speck, A. K. The Mineralogy of Dust Around Evolved Stars 1998, PhD Thesis.

[6] Marra, A.C., Lane, M.D., Orofino, C., Blanco, A., Fonti, S., Midinfrared spectra and optical constants of bulk hematite: Comparison with particulate hematite spectra 2011, Icarus, 211, 839.

[7] Corman, A.B. Carbon Stars and Silicon Carbide 2010, Ph.D. Thesis, University of Missouri

[8] Pegourie, B. Optical properties of alpha silicon carbide 1988, A\&A, 194, 335. 
[9] Min, M., Hovenier, J. W., de Koter, A., Shape effects in scattering and absorption by randomly oriented particles small compared to the wavelength 2003, A\&A, 404, 35.

[10] Speck, A.K., Hofmeister, A.M., Barlow, M.J., The SiC Problem: Astronomical and Meteoritic Evidence 1999, ApJL, 513, 87.

[11] Gillett, F. C., Low, F. J., Stein, W. A. Stellar Spectra from 2.8 to 14 Microns 1968, ApJ, 154, 677.

[12] Knacke, R. F., Gaustad, J. E., Gillett, F. C., Stein, W. A. A Possible Identification of Interstellar Silicate Absorption in the Infrared Spectrum of 119 Tauri 1969, ApJL, 155, L189.

[13] Hackwell, J. A., Gehrz, R. D., Woolf, N. J. Interstellar Silicate Absorption Bands 1970, Nature, 227, 822.

[14] Mann, Ingrid, Köhler, Melanie, Kimura, Hiroshi, Cechowski, Andrzej, Minato, Tetsunori, Dust in the solar system and in extra-solar planetary systems 2006, A\&ARev, 13, 159.

[15] Speck, A. K., Barlow, M. J., Sylvester, R. J., Hofmeister, A. M. Dust features in the 10- $\mu$ m infrared spectra of oxygen-rich evolved stars 2000, A\&AS, 146, 437.

[16] Casassus, S., Roche, P. F., Aitken,D. K., Smith, C. H. The Galactic disc distribution of planetary nebulae with warm dust emission features 2001, MNRAS, 320, 424.

[17] Chiar, J. E., Tielens, A. G. G. M. Pixie Dust: The Silicate Features in the Diffuse Interstellar Medium 2006, ApJ, 637, 774.

[18] Chiar, J. E., Ennico, K., Pendleton, Y. J.The Relationship between the Optical Depth of the $9.7 \mu \mathrm{m}$ Silicate Absorption Feature and Infrared Differential Extinction in Dense Clouds 2007, ApJ, 666, L73.

[19] Hao, Lei, Spoon, H. W. W., Sloan, G. C., et al. The Detection of Silicate Emission from Quasars at 10 and 18 Microns 2005, ApJ, 625, L75.

[20] Woolf, N. J., Ney, E. P. Circumstellar Infrared Emission from Cool Stars 1969, ApJ, 155, L181.

[21] Gaustad, John E. The Opacity of Diffuse Cosmic Matter and the Early Stages of Star Formation, 1963, ApJ, 138, 1050.

[22] Gilman, R.C. On the Composition of Circumstellar Grains 1969, ApJ, 155, L185.

[23] Woolf, N. J. Circumstellar Infrared Emission. I the Circumstellar Origin of Interstellar Dust, 1973 "Interstellar Dust and Related Topic"s. IAU Symp. 52, (Eds) J. Mayo Greenberg and H. C. van de Hulst. Dordrecht, Boston, Reidel, p.485

[24] Huffman, D. R., Stapp, J. L. Optical Measurements on Solids of Possible Interstellar Importance 1973, in “Interstellar Dust and Related Topics. IAU Symp. 52," (Eds) J. Mayo Greenberg and H. C. van de Hulst. Dordrecht, Boston, Reidel, p.297.

[25] Pollack, J. B., Toon, O. B., Khare, B. N. Optical properties of some terrestrial rocks and glasses 1973, Icarus, 19, 372.

[26] Day, K.L. Mid-infrared optical properties of vapor-condensed magnesium silicates 1979, ApJ, 234, 158.

[27] Krätschmer, W., Huffman, D. R. Infrared extinction of heavy ion irradiated and amorphous olivine, with applications to interstellar dust 1979, Astrophys. Sp. Sci., 61, 195.

[28] Draine, B. T., Lee, H. M. Optical properties of interstellar graphite and silicate grains 1984, ApJ, 285, 89.

[29] Ossenkopf, V., Henning, Th., \& Mathis, J. S. Constraints on cosmic silicates 1992, A\&A, $261,567$. 
[30] Draine, B.T., Interstellar Dust Grains, 2003, ARA\&A, 41, 241.

[31] Nuth, J. A., III, Donn, B. Laboratory measurements of amorphous silicate smokes and the infrared spectra of oxygen-rich stars 1982, ApJ, 257, L103

[32] Scott, A., Duley, W. W. Ultraviolet and Infrared Refractive Indices of Amorphous Silicates 1996, ApJS, 105, 401.

[33] Dorschner, J., Begemann, B., Henning, T., Jaeger, C., Mutschke, H. Steps toward interstellar silicate mineralogy. II. Study of Mg-Fe-silicate glasses of variable composition 1995, A\&A, 300, 503.

[34] Jäger, C., Dorschner, J., Mutschke, H., Posch, Th., Henning, Th. Steps toward interstellar silicate mineralogy. VII. Spectral properties and crystallization behaviour of magnesium silicates produced by the sol-gel method 2003, A\&A, 408, 193.

[35] Speck, A. K., Whittington, A. G., Hofmeister, A. M., Disordered Silicates in Space: A Study of Laboratory Spectra of "Amorphous" Silicates 2011, ApJ, 74093.

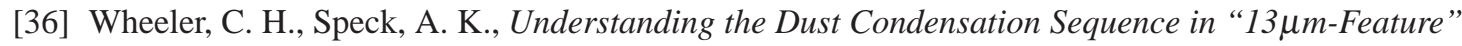
Oxygen-Rich AGB Stars Using Radiative Transfer Models 2007, BAAS, 39, 890.

[37] Sargent, B., Srinivasan, S., Meixner, M., et al., The Mass-loss Return from Evolved Stars to the Large Magellanic Cloud. II. Dust Properties for Oxygen-rich Asymptotic Giant Branch Stars 2010, ApJ, 716, 878.

[38] Volk, K., Kwok, S., Spectral evolution of asymptotic giant branch stars 1988, ApJ, 331, 435.

[39] Mennella, V., Brucato, J. R., Colangeli, L.; Palumbo, P., Rotundi, A., Bussoletti, E., Temperature Dependence of the Absorption Coefficient of Cosmic Analog Grains in the Wavelength Range 20 Microns to 2 Millimeters 1998, ApJ, 4961058.

[40] Koike, C., Hasegawa, H., Manabe, A., Extinction coefficients of amorphous carbon grains from 2100 A to 340 microns, 1980, Ap\&SS 67, 495.

[41] Speck, A. K., Buffard, A. Pitman, K., Hofmeister, A. M., Whittington, A. G., Better Alternatives to "Astronomical Silicate": Laboratory-Based Optical Functions of Glass With Chondritic/Solar Abundances With Application to HD 1617962014 , to be submitted to ApJ. 